\title{
Extending the detection limit of dopants for focused ion beam prepared semiconductor specimens examined by off-axis electron holography
}

\author{
Cooper, David; Rivallin, Pierrette; Hartmann, Jean-Michel; Chabli, Amal; Dunin-Borkowski, Rafal E.
}

Published in:

Journal of Applied Physics

Link to article, DOI:

$10.1063 / 1.3195088$

Publication date:

2009

Document Version

Publisher's PDF, also known as Version of record

Link back to DTU Orbit

Citation (APA):

Cooper, D., Rivallin, P., Hartmann, J-M., Chabli, A., \& Dunin-Borkowski, R. E. (2009). Extending the detection limit of dopants for focused ion beam prepared semiconductor specimens examined by off-axis electron holography. Journal of Applied Physics, 106(6), 064506. https://doi.org/10.1063/1.3195088

\section{General rights}

Copyright and moral rights for the publications made accessible in the public portal are retained by the authors and/or other copyright owners and it is a condition of accessing publications that users recognise and abide by the legal requirements associated with these rights.

- Users may download and print one copy of any publication from the public portal for the purpose of private study or research.

- You may not further distribute the material or use it for any profit-making activity or commercial gain

- You may freely distribute the URL identifying the publication in the public portal 


\title{
Extending the detection limit of dopants for focused ion beam prepared semiconductor specimens examined by off-axis electron holography
}

\author{
David Cooper, ${ }^{1, a)}$ Pierrette Rivallin, ${ }^{1}$ Jean-Michel Hartmann, ${ }^{1}$ Amal Chabli, ${ }^{1}$ and \\ Rafal E. Dunin-Borkowski ${ }^{2}$ \\ ${ }^{1}$ CEA, LETI, MINATEC, 17 rue des martyrs, F38054 Grenoble Cedex 9, France \\ ${ }^{2}$ Centre for Electron Nanoscopy, Technical University of Denmark, DK-2800 Kongens Lyngby, Denmark
}

(Received 15 May 2009; accepted 9 July 2009; published online 21 September 2009)

\begin{abstract}
Silicon specimens containing $p-n$ junctions have been prepared for examination by off-axis electron holography using focused ion beam (FIB) milling. FIB milling modifies the surfaces of the specimens due to gallium implantation and the creation of defects which has the effect of reducing the active dopant concentration measured during electrical characterization. Here we show that although this damage can be removed by using low temperature annealing, the presence of surface charge will modify the electrical potentials in the specimens and limit the dopant concentration that can be measured. (c) 2009 American Institute of Physics. [doi:10.1063/1.3195088]
\end{abstract}

\section{INTRODUCTION}

The nanoscale dimensions of state-of-the-art semiconductor devices have placed increasing demands on the techniques that are required to provide maps of the dopants in these devices. ${ }^{1}$ Currently, techniques that are under development for dopant profiling include off-axis electron holography, ${ }^{2}$ scanning electron microscopy (SEM), ${ }^{3}$ scanning spreading resistance microscopy (SSRM), ${ }^{4}$ and scanning capacitance microscopy (SCM). ${ }^{5}$ The key to success for all of these techniques is specimen preparation using a method that can find the region of interest in nanometer-scale devices. Currently, focused ion beam (FIB) milling is the only technique that has the required site specificity and the success rate which is required to prepare these specimens for characterization. FIB milling typically uses $30 \mathrm{kV}$ Ga ions to extract the region of interest from a wafer, and in the case for observations using transmission electron microscopy (TEM) to then thin the specimen to electron transparency. Silicon specimens prepared using a FIB operated at $30 \mathrm{kV}$ will typically have an amorphous surface layer of around $25 \mathrm{~nm}$ and then a near-surface layer containing defects extending to more than $100 \mathrm{~nm}$ in depth which can trap the dopants that are present in this region. ${ }^{6-8}$ Therefore the effects of FIB milling will significantly influence the results for dopant profiling techniques that are performed in transmission such as electron holography and will make surface scanning techniques such as SSRM, SCM, and SEM extremely difficult. Although low energy FIB milling ${ }^{9}$ and/or additional techniques such as low energy Ar ion milling ${ }^{10}$ can be used to improve the specimens, ${ }^{11}$ recent experimental results suggest that although the damaged regions can be reduced, they are not removed. ${ }^{12,13}$ In this study we have sought to understand the effects and then remove the damage that is introduced in semiconductor specimens during FIB preparation.

Off-axis electron holography has been used to characterize a series of $p-n$ junction specimens prepared using FIB milling. Off-axis electron holography is a TEM based tech-

${ }^{a)}$ Electronic mail: david.cooper@cea.fr. nique that uses a biprism to interfere an object wave that has passed through a specimen, with a reference wave that has passed through only a vacuum. From the resulting interference pattern which is also known as a hologram, both phase and amplitude images of the specimen can be reconstructed. ${ }^{2}$ When no magnetic fields or diffraction contrast is present, the change in phase of an electron as it passes through a specimen can be given by the expression

$$
\Delta \phi=C_{E} \int_{0}^{t} V(x, y, z) d z,
$$

where $C_{E}$ is a constant dependent on the energy of the electron wave $\left(7.29 \times 10^{-3} \mathrm{rad} \mathrm{V}^{-1} \mathrm{~nm}^{-1}\right.$ for $200 \mathrm{kV}$ electrons), $V$ is the electrostatic potential, and $z$ is the electron beam direction. ${ }^{14}$ The phase of an electron is very sensitive to changes in potential in a specimen such as from the presence of dopants. Therefore in principle, electron holography can be used to fulfill the requirements of the semiconductor industry for a quantitative dopant profiling technique with nanometer-scale resolution. ${ }^{15}$

In order to determine the effects of FIB milling on $\mathrm{Si}$ semiconductor devices, a series of symmetrically doped $p-n$ or $n-p$ junctions have been grown using reduced-pressure chemical vapor deposition. For each of the junctions a $1.0 \mu \mathrm{m}$ layer of phosphorus ( $n$-type) doped $\mathrm{Si}$ was grown onto a $1.0 \mu \mathrm{m}$ layer of boron ( $p$-type) doped $\mathrm{Si}$ onto a lightly $p$-doped substrate (or vice versa in the case of the $p-n$ junctions). Secondary ion mass spectrometry was performed on each of the specimens to measure the dopant concentrations. These were found to be $2 \times 10^{17}, 2 \times 10^{18}$, and $1 \times 10^{19} \mathrm{~cm}^{-3}$. Parallel-sided specimens were prepared for examination by off-axis electron holography from each of the differently doped wafers by using the FIB operated at either 30 or $8 \mathrm{kV}$ using a conventional trench geometry. ${ }^{8,16}$ Prior to milling, a metallic coating was sputtered onto the surfaces of the specimens. Then a $1.0-\mu \mathrm{m}$-thick protective tungsten layer was deposited directly over the region of in- 


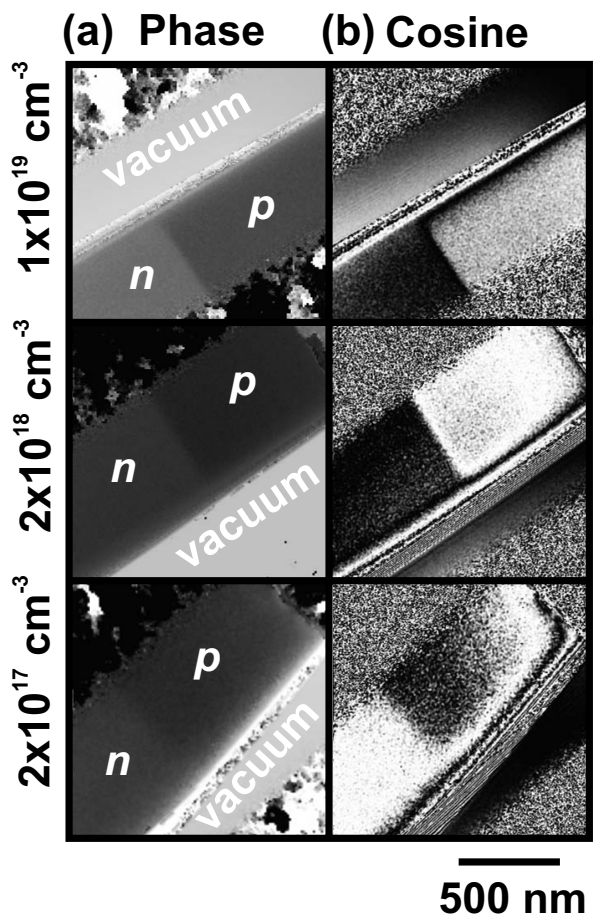

FIG. 1. (a) Phase images for 400-nm-thick $p$ - $n$ junctions with the different dopant concentrations. (b) Cosine of the phase clearly showing that the near-surface regions of the specimens are at a negative ( $n$-type) potential.

terest using ion beam assisted deposition in order to prevent normal incidence ion implantation and milling from the tails of the beam.

Holograms of the specimens were acquired using a probe corrected field emission gun FEI Titan microscope operated at $200 \mathrm{kV}$ (although the probe corrector was not used). The objective lens and third condenser lens were turned off, and a Lorentz lens was used to extend the holographic field of view to $1500 \times 700 \mathrm{~nm}^{2}$. The fringe spacing in the holograms was $3.5 \mathrm{~nm}$ which provided a spatial resolution of $\sim 10 \mathrm{~nm}$ in the reconstructed phase images. It is possible to achieve a much better spatial resolution than this, ${ }^{17}$ however, here the experiment has been optimized to maximize the field of view. In addition the stability of the state-of-the-art electron microscope allowed holograms to be acquired using a low beam intensity and for long time periods in order to reduce both specimen charging and improve the signal-tonoise ratio. ${ }^{18}$

\section{OFF-AXIS ELECTRON HOLOGRAPHY \\ OF $P$ - $N$ JUNCTIONS WITH DIFFERENT DOPANT CONCENTRATIONS AND LOW TEMPERATURE IN SITU ANNEALING}

Figure 1 shows reconstructed phase images acquired for specimens containing the differently doped $p$ - $n$ junctions. All of the specimens have an approximate crystalline thickness of $400 \mathrm{~nm}$ which was measured using convergent beam electron diffraction (CBED). The differently doped regions are clearly visible. The cosine of the phase is also shown. The $p-n$ junctions in the phase images clearly do not extend all the way to the specimen surface. This is particularly the case for the specimens which have lower dopant concentrations. Another observation from the phase images is that the nearsurface regions appear to be at the same potential as the $n$-doped layers in the junctions. Observations of more than 20 specimens (each with around five membranes of different thicknesses) containing either $p-n$ or $n-p$ junctions have shown that the surfaces of the specimens tend to be at the same potential as the doped regions that are directly attached to the substrate. However, we note that for just one of these specimens (which contained five different membranes), the surfaces were observed to be at the same potential as the $n$-type surface layer.

A final observation is that the electrostatic fringing fields that would be expected to be seen originating from the $p$ - $n$ junctions in the vacuum regions are not observed. A slight gradient can be seen in the vacuum in the specimen with the dopant concentration of $1 \times 10^{19} \mathrm{~cm}^{-3}$; however, this clearly does not originate from the junction.

In order to quantify the step in phase measured across the junctions, phase profiles were extracted from across a series of specimens containing $p$ - $n$ junctions of different thicknesses. It is known that during FIB preparation, defects will be introduced deep in the crystalline regions of the specimens which has the effect of trapping the dopants. ${ }^{7}$ This artifact is one of the principal mechanisms of what is referred to as the electrically inactive layer. This inactive layer can be observed both in Fig. 1 where the junctions do not extend to the specimen surfaces and in Fig. 2. Figure 2 shows the step in phase measured across the different specimens as a function of the crystalline specimen thickness measured using CBED. The $x$-intercept for each of the data sets gives a value

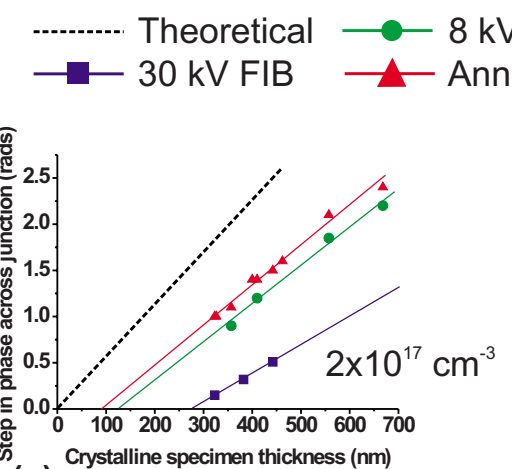

(a)

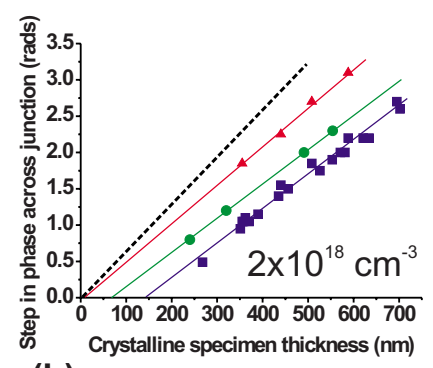

(b)

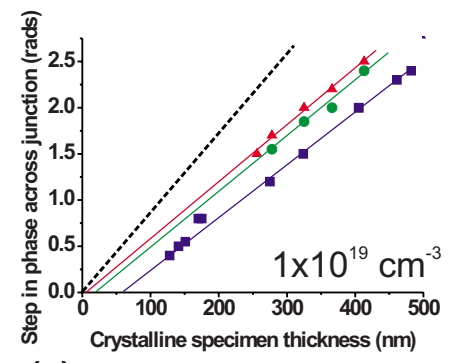

(c)

FIG. 2. (Color online) The step in phase measured across the $p-n$ junctions as a function of the crystalline specimen thickness. The specimens have been examined after FIB preparation at 30 and $8 \mathrm{kV}$ and after annealing in vacuum. (a), (b), and (c) refer to the specimens with dopant concentrations of 2 $\times 10^{17}, 2 \times 10^{18}$, and $1 \times 10^{19} \mathrm{~cm}^{-3}$, respectively. 
TABLE I. The electrically inactive thickness measured (in units of nanometers) for each of the differently doped specimens for each of the different preparation methods. The annealed specimens can be prepared using either 30 or $8 \mathrm{kV} \mathrm{Ga}$ ions to achieve the same experimental result. The experimental error in each case is $\pm 15 \mathrm{~nm}$.

\begin{tabular}{cccc}
\hline \hline $\begin{array}{c}\text { Dopant concentration } \\
\left(\mathrm{cm}^{-3}\right)\end{array}$ & $30 \mathrm{kV}$ & $8 \mathrm{kV}$ & Annealed at $350{ }^{\circ} \mathrm{C}$ \\
\hline $1 \times 10^{19}$ & 60 & 25 & 5 \\
$2 \times 10^{18}$ & 140 & 60 & 10 \\
$2 \times 10^{17}$ & 250 & 13 & 85 \\
\hline \hline
\end{tabular}

for the electrically inactive thickness. In this crystalline layer, none of the dopants that are present contribute toward the measured potential and their presence leads to a measurement of the potential across the $p$ - $n$ junctions which is much less than predicted by theory. A clear definition of the inactive thickness, $t_{\text {inactive }}$ is the total crystalline thickness on both faces of the specimen that is altered during preparation, where $t_{\text {inactive }} / 2$ is present on each specimen surface. As the specimen thickness is measured by CBED, the thickness of the amorphous layers is not included.

It has been shown recently that the electrically inactive thickness is strongly dependent on the dopant concentration in the specimens, ${ }^{8}$ therefore this artifact cannot be accounted for simply by subtracting a fixed value from the total crystalline thickness. It is known that the electrically inactive thickness can be reduced either by reducing the operating voltage of the $\mathrm{FIB}^{12}$ or by performing a low temperature anneal on the specimens. ${ }^{7}$ To assess these improvements as a function of the dopant concentration, specimens were prepared using the FIB operated at either 30 or $8 \mathrm{kV}$ and electron holograms were acquired. The specimens prepared at each FIB operating voltage were then annealed in situ in a plasma cleaner (with the plasma off) for $30 \mathrm{~min}$ at $350{ }^{\circ} \mathrm{C}$ using a Gatan heating holder. At these low temperatures, the dopant diffusion observed in these specimens will be less than $1 \mathrm{~nm} ;{ }^{7}$ however, it should be considered that the diffusion could be higher in implanted complementary metaloxide semiconductor devices. Figures 2(a)-2(c) show the step in phase for the differently doped $p$ - $n$ junctions as a function of the crystalline specimen thickness. For all of the specimens the electrically inactive thickness is found to be reduced by a factor of 2 when the FIB operating voltage is reduced from 30 to $8 \mathrm{kV}$ which is consistent with previous observations of GaAs $p$ - $n$ junctions. ${ }^{19}$ After the low temperature annealing the electrically inactive thickness has been almost completely removed for the specimens with dopant concentrations of $2 \times 10^{18}$ and $1 \times 10^{19} \mathrm{~cm}^{-3}$. However, for the specimen with the dopant concentration of $2 \times 10^{17} \mathrm{~cm}^{-3}$, a large inactive thickness remains, even after multiple attempts to remove it using specimens prepared at different times, using different FIB operating voltages and different annealing temperatures up to $500{ }^{\circ} \mathrm{C}$.

Table I shows the measured values of the electrically inactive thickness for each of the different specimens for each method of preparation. From the table it is clear that the inactive thickness is dependent on both the preparation method and dopant concentrations in the specimens.
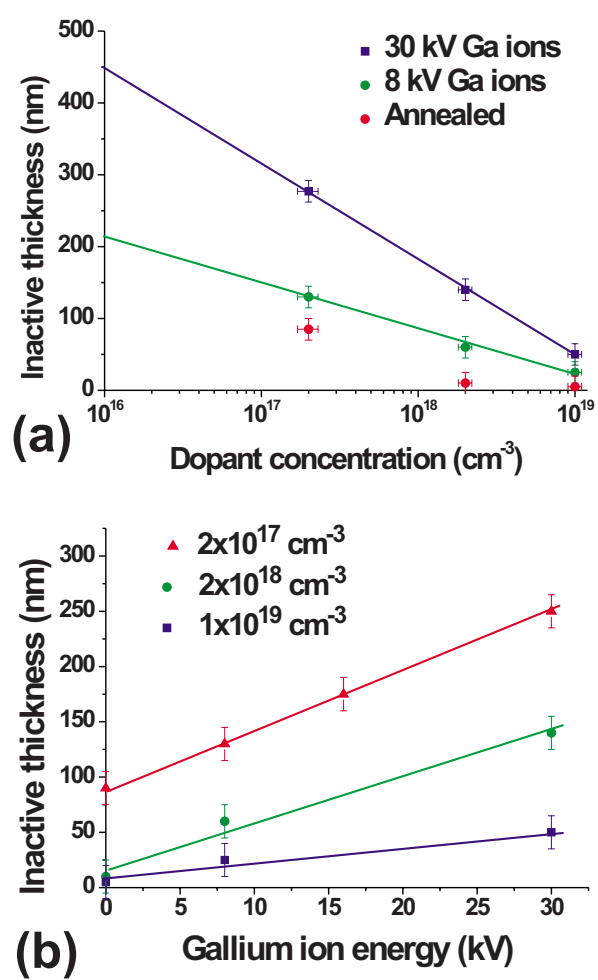

FIG. 3. (Color online) (a) The electrically inactive thickness as a function of the dopant concentration in the specimens. (b) The electrically inactive thicknesses measured in the differently doped specimens as a function of the FIB operating voltage. Specimens that were annealed at low temperature have been indicated as having been prepared at $0 \mathrm{kV}$.

Also, the built-in potential $V_{\mathrm{bi}}$ in the $p-n$ junction can be measured from the gradient,

$$
V_{\mathrm{bi}}=\frac{1}{C_{E}}\left(\frac{\Delta \phi}{t_{\text {crystalline }}-t_{\text {inactive }}}\right) \text {, }
$$

where $t_{\text {crystalline }}$ is the crystalline thickness of the specimens and $t_{\text {inactive }}$ is the electrically inactive thickness. Although this method of measuring $V_{\mathrm{bi}}$ is in principle independent of the inactive thickness, in Figs. 2(a)-2(c), it can be seen that the values measured experimentally from the gradients are not consistent with the expected values that are indicated by the dashed line. ${ }^{20}$ Reducing the electrically inactive thickness does not significantly increase the measured values of $V_{\mathrm{bi}}$ in the junctions. It has been shown experimentally that the reasons for this difference are due to the specimen charging during TEM examination. This has been described in more detail elsewhere. ${ }^{8,13,21}$

Figure 3(a) shows the experimentally measured inactive thickness as a function of the dopant concentration in the specimens. Assuming that FIB damage is the only cause of the inactive layer, the lower limit of the dopant concentration that can be detected using off-axis electron holography can be determined by extrapolating the gradient to the $y$-intercept. For example, for specimens prepared using $30 \mathrm{kV}$ Ga ions, a crystalline specimen thickness of more than $450 \mathrm{~nm}$ will be required to detect a dopant concentration of $1 \times 10^{16} \mathrm{~cm}^{-3}$. The graph also suggests that this detection limit can be improved by operating the FIB at $8 \mathrm{kV}$. In this 


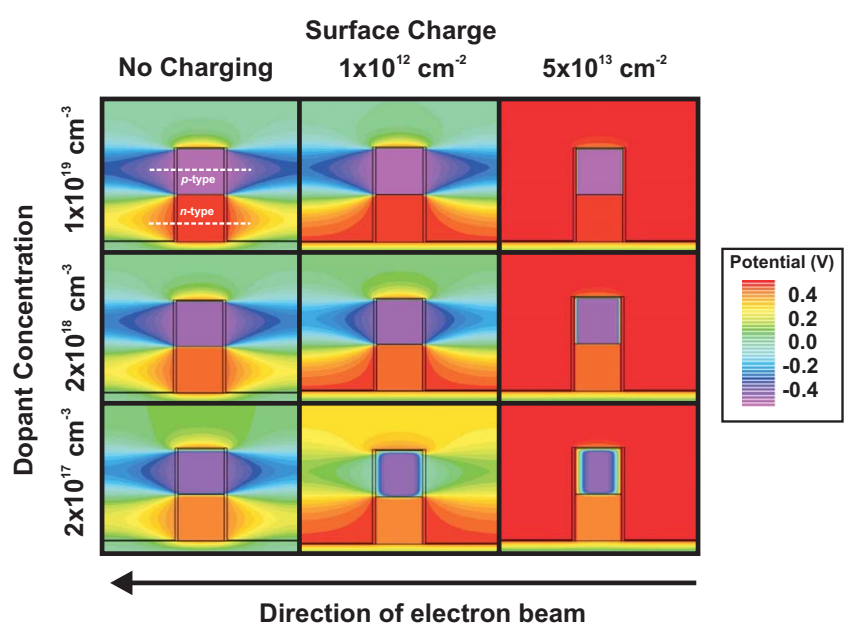

FIG. 4. (Color online) 2D simulations of the potential in specimens with dopant concentrations of $2 \times 10^{17}, 2 \times 10^{18}$, and $1 \times 10^{19} \mathrm{~cm}^{-3}$. The specimens are assumed to be perfect in the simulations with a 25 -nm-thick amorphous layer present on each surface. A layer of charge has been placed between the crystalline and amorphous regions and has been varied to assess the effects of the potentials within the differently doped regions.

instance, a specimen thickness of just over $220 \mathrm{~nm}$ would be required to detect a concentration of $1 \times 10^{16} \mathrm{~cm}^{-3}$.

However, the effects of charge states at the specimen surfaces will also contribute toward the inactive thickness. Figure 3(b) shows the electrically inactive thickness measured experimentally in the specimens as a function of the FIB operating voltage. The specimens that have been annealed are indicated as being prepared using the FIB operated at $0 \mathrm{kV}$. The intercept with the $y$-axis indicates the electrically inactive thickness that remains in perfect, crystalline TEM specimens due to surface depletion. The surface depletion will be caused by charged states on the specimen surfaces. $^{22,23}$ The experimental data shown in Fig. 3(b) suggest that the effects from the charged surfaces are slight for specimens with high dopant concentrations but are significant for the specimen observed here with a concentration of $2 \times 10^{17} \mathrm{~cm}^{-3}$.

\section{SIMULATIONS}

To help understand the effects of surface depletion, two dimensional (2D) simulations were performed using the commercially available potential simulator ATLAS by Silvaco. ${ }^{24}$ The potentials in the specimens were calculated using Poisson's equation. A 300-nm-thick $p-n$ junction was created with a 20-nm-thick amorphous layer and a layer of charge in between the crystalline and amorphous regions. The values of the surface charges were then varied to see the effects on the potential inside the specimens.

Figure 4 shows 2D potential profiles for the different specimens. Negative values of surface charge were used in the simulations as the phase images in Fig. 1 suggest that the surfaces are at a similar potential to the $n$-doped regions in the specimens. From the simulations it is evident that when no charge is present on the specimen surfaces, strong fringing fields are observed in the vacuum regions of the specimen originating from the $p-n$ junction. These fields have

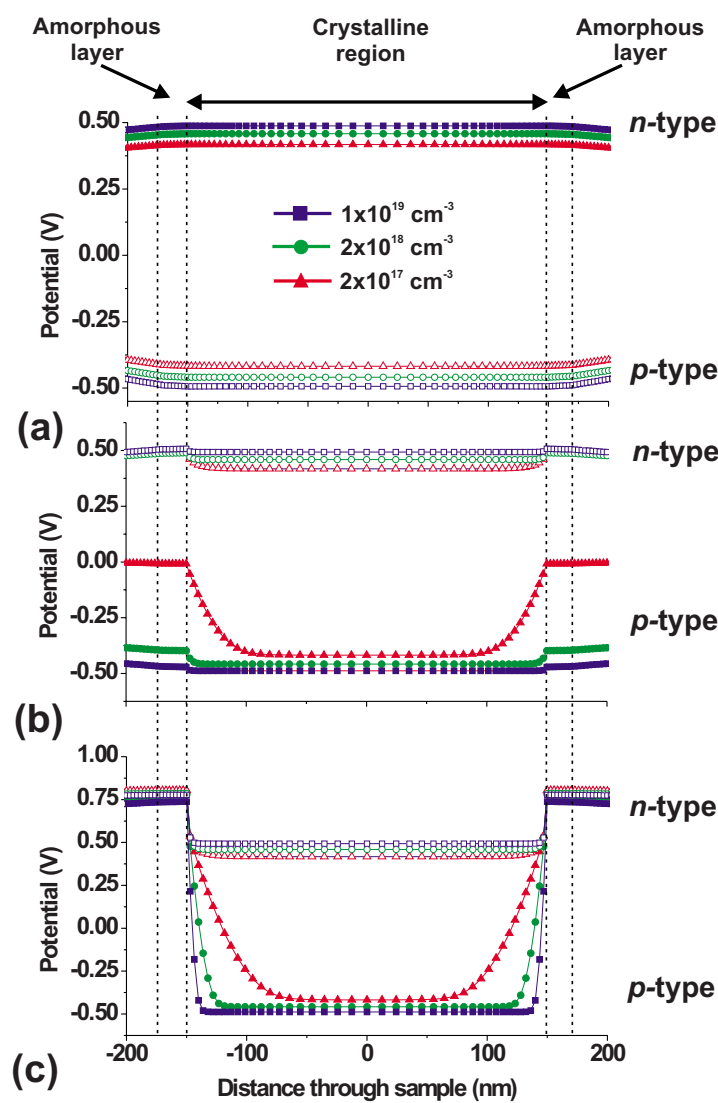

FIG. 5. (Color online) Potential profiles extracted from across the simulations of the differently doped $p$ - $n$ junctions for sheet charges of (a) 0 , (b) $1 \times 10^{12}$, and $(\mathrm{c}) 5 \times 10^{13} \mathrm{~cm}^{-2}$.

never been observed in FIB-prepared specimens, although they have been seen in cleaved Si $p$ - $n$ junctions with relatively high dopant concentrations. ${ }^{25}$

The minimum value of surface charge required to remove the fringing fields from the most highly doped samples was found to be $5 \times 10^{13} \mathrm{~cm}^{-2}$. This value is close to the values of charge that have been used previously to fit experimental data to simulations of $p-n$ junctions. ${ }^{22}$ When this surface charge is applied to all of the specimens, a relatively small but significant amount of band bending is observed in the $p$-type regions of the highly doped specimens. However, in the specimens with a dopant concentration of $2 \times 10^{17} \mathrm{~cm}^{-3}$ there are significant surface effects which explain the large residual electrically inactive thickness that is observed in the annealed TEM specimens. Simulations are also shown using an intermediate surface charge of $1 \times 10^{12} \mathrm{~cm}^{-2}$ to demonstrate that in this case, fringing fields remain in the vacuum.

Figures 5(a)-5(c) show profiles extracted from across the $p$ and $n$ doped regions in the direction of the electron beam for each of the differently doped specimens with each different surface charge. Figure 5(a) shows the profiles with no charge applied to the surfaces, with $1 \times 10^{12} \mathrm{~cm}^{-2}$ [Fig. 5(b)], and with $5 \times 10^{13} \mathrm{~cm}^{-2}$ [Fig. 5(c)]. These profiles were extracted from regions such as the one indicated by the dashed lines in Fig. 4. The profiles were acquired well away from the region of the $p-n$ junction to assess only the effects of surface charging. 
TABLE II. The electrically inactive thicknesses (in units of nanometers) after annealing in the differently doped specimens measured experimentally and from simulations using either a sheet charge of $5 \times 10^{13} \mathrm{~cm}^{-2}$ on the specimen surface or a 25 -nm-thick layer containing a positive charge (volume) of $2 \times 10^{18} \mathrm{~cm}^{-3}$ layers. The error in the experimental profiles is \pm 15 nm.

\begin{tabular}{cccc}
\hline \hline $\begin{array}{c}\text { Dopant concentration } \\
\left(\mathrm{cm}^{-3}\right)\end{array}$ & $\begin{array}{c}\text { Expt. } \\
(\mathrm{nm})\end{array}$ & $\begin{array}{c}\text { Sheet charge } \\
(\mathrm{nm})\end{array}$ & $\begin{array}{c}\text { Volume charge } \\
(\mathrm{nm})\end{array}$ \\
\hline $1 \times 10^{19}$ & 5 & 35 & 15 \\
$2 \times 10^{18}$ & 10 & 65 & 25 \\
$2 \times 10^{17}$ & 85 & 95 & 90 \\
\hline \hline
\end{tabular}

From the profiles it is clear that the $p$-type regions in all of the junctions are modified due to the presence of negative charge on the surfaces. By integrating the potential from the profiles, the inactive thickness can be calculated from the simulations. Table II shows the experimentally determined values of the inactive thickness in specimens that are assumed to be "perfect" after annealing. Also shown are the inactive thicknesses derived from simulations using a sheet charge of $5 \times 10^{13} \mathrm{~cm}^{-2}$. Although the simulations can account for the absence of fringing fields in vacuum, they also suggest that a large inactive thickness will be present for all of the differently doped specimens examined here.

In order to obtain a better fit between the simulations and experimental data the simulations were modified. Instead of using a surface charge, it was assumed that the amorphous layer could trap charge. In the simulations a 5- or 20-nmthick layer with a volume charge of $2 \times 10^{18} \mathrm{~cm}^{-3}$ was required to remove the fringing fields from the vacuum for all of the differently doped specimens. The two different thicknesses of the layer were selected as specimens prepared using FIB milling at either 8 or $30 \mathrm{kV}$ are expected to have amorphous layers on each face of approximately this thickness. No difference was seen between the simulated inactive thicknesses for the specimens with the different thickness amorphous layers. Profiles extracted from across the different junctions are shown in Fig. 6. The values of the inactive thickness calculated from this model are also shown in Table II. Although this improved model gives a much better fit between the experimentally measured values and the simulations, the model used here overestimates the inactive thickness for the specimens with the higher dopant concentra-

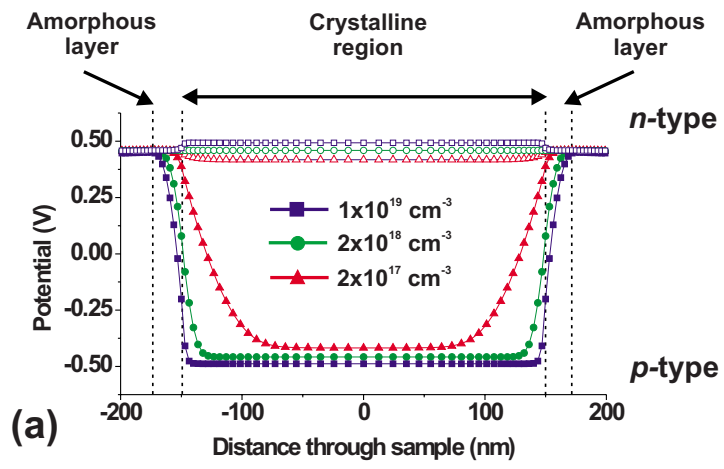

FIG. 6. (Color online) Potential profiles extracted from across the simulations for the differently doped $p$ - $n$ junctions with $25-\mathrm{nm}$-thick amorphous layers containing a trapped negative charge of $2 \times 10^{18} \mathrm{~cm}^{-3}$.

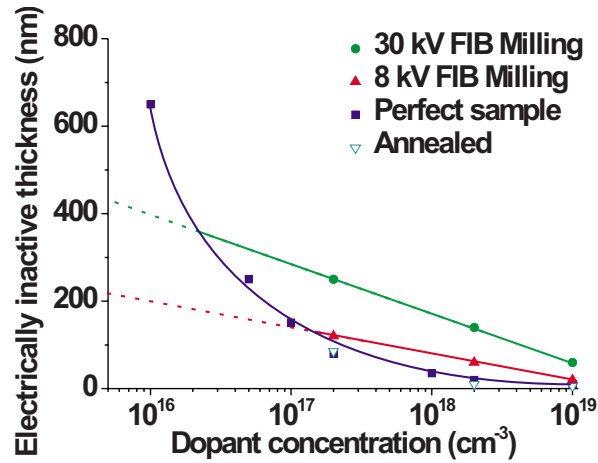

FIG. 7. (Color online) The electrically inactive thickness as a function of dopant concentration in symmetrical $p$ - $n$ junctions for simulated perfect specimens and for specimens prepared using a FIB operated at both 30 and $8 \mathrm{kV}$ and measured experimentally. The inactive thicknesses for the annealed specimens are also shown.

tions. However, the fit is just within experimental error and therefore can provide a reasonable estimation of the effects of band bending in doped specimens.

\section{SUMMARY}

It has been shown that in FIB-milled specimens containing $p-n$ junctions there is an inactive thickness that will result in the measured potentials being much less than predicted by theory. This layer is also dependent on the dopant concentration and the specimen preparation method employed. This inactive thickness has been almost completely removed by annealing the specimens at low temperature to remove the defects that are introduced during FIB preparation for dopant concentrations of $2 \times 10^{18} \mathrm{~cm}^{-3}$ or more. However, in the specimen examined with a dopant concentration of $2 \times 10^{17} \mathrm{~cm}^{-3}$ a large inactive thickness is present, even after annealing.

Simulations have shown that the presence of fringing fields in vacuum can be eliminated by using a sheet charge of $5 \times 10^{13} \mathrm{~cm}^{-2}$ on the specimen surfaces. However, the presence of this charge will lead to large inactive thicknesses in all of the specimens, which is not observed experimentally. By putting $2 \times 10^{19} \mathrm{~cm}^{-3}$ of trapped charge in either a 5- or 20-nm-thick amorphous layer on each of the specimen surfaces, both the external fringing fields can be removed and the experimental data reproduced to within error.

By using these values of charge, a lower limit of dopant detection can be calculated. Figure 7 shows the inactive thickness for a range of differently doped specimens, obtained both from simulations and experiment, using values from specimens prepared using a FIB operated at both 30 and $8 \mathrm{kV}$. Here it can be seen that the inactive thickness that is introduced during FIB milling is the dominant component of the inactive thickness down to a dopant concentration of $2 \times 10^{16} \mathrm{~cm}^{-3}$ for specimens prepared using $30 \mathrm{kV}$ Ga ions and $2 \times 10^{17} \mathrm{~cm}^{-3}$ for specimens prepared using $8 \mathrm{kV} \mathrm{Ga}$ ions. At dopant concentrations lower than these, the effects of charging on the specimen surface will influence the inactive thickness. The simulations suggest that due to the effects 
of surface charging, it is unlikely that dopant concentrations below $1 \times 10^{16} \mathrm{~cm}^{-3}$ can be detected as the specimen thicknesses above $600 \mathrm{~nm}$ would be required.

\section{CONCLUSION}

Electron holograms have been acquired for Si specimens containing $p-n$ junctions with different dopant concentrations. The specimens have been prepared using a FIB operated at different voltages. From the reconstructed phase images an electrically inactive thickness has been identified which is dependent on both the dopant concentration in the specimens and the FIB operating voltage. The specimens have then been annealed at low temperature in order to remove the defects that are introduced during FIB milling. After the anneal, the inactive thickness has been almost completely eliminated for the dopant concentrations above $2 \times 10^{18} \mathrm{~cm}^{-3}$; however, it remains significant in specimens with lower dopant concentrations. By comparing the experimental data with simulations of the $p-n$ junctions, it has been shown that a negative charge of $2 \times 10^{18} \mathrm{~cm}^{-3}$ in the amorphous layer is required to make the best fit. This value is consistent with expected values of trapped charge in $\mathrm{Si}-\mathrm{SiO}_{2}$ interfaces. $^{20}$

The results that have been obtained here using off-axis electron holography have been shown to be reproducible if sufficient care is taken during specimen preparation and during examination in the TEM. ${ }^{8}$ By understanding the artifacts that are introduced into FIB-milled specimens that have been either prepared at different energies or annealed, we hope to be able to reliably fit the results to simulations that can provide quantitative information about the dopant concentrations in semiconductor specimens.

\section{ACKNOWLEDGEMENTS}

This work was partly funded by the French Public Authorities through the NANO 2012 program.
${ }^{1}$ M. A. Castell, D. A. Muller, and P. M. Voyles, Nature (London) 2, 129 (2003).

${ }^{2}$ M. R. McCartney and D. J. Smith, Annu. Rev. Mater. Res. 37, 729 (2007). ${ }^{3}$ P. Kazemian, A. C. Twitchett, C. J. Humphreys, and C. Rodenburg, Appl. Phys. Lett. 88, 212110 (2006).

${ }^{4}$ P. Eyben, D. Alvarez, M. Jurczak, R. Rooyackers, A. De Keersgieter, E. Augendre, and W. Vandervorst, J. Vac. Sci. Technol. B 22, 364 (2004).

${ }^{5}$ C. C. Williams, Annu. Rev. Mater. Sci. 29, 471 (1999).

${ }^{6}$ A. C. Twitchett, R. E. Dunin-Borkowski, R. J. Hallifax, R. F. Broom, and P. A. Midgley, Phys. Rev. Lett. 88, 238302 (2002).

${ }^{7}$ D. Cooper, A. C. Twitchett, P. K. Somodi, I. Farrer, D. A. Ritchie, P. A. Midgley, and R. E. Dunin-Borkowski, Appl. Phys. Lett. 88, 063510 (2006).

${ }^{8}$ D. Cooper, C. Ailliot, R. Truche, J. Hartmann, J. Barnes, and F. Bertin, J. Appl. Phys. 104, 064513 (2008).

${ }^{9}$ N. I. Kato, Y. Kohno, and H. Saka, J. Vac. Sci. Technol. A 17, 1201 (1999).

${ }^{10}$ A. Barna, B. Pecz, and M. Menyhard, Ultramicroscopy 70, 161 (1998).

${ }^{11}$ M. R. McCartney, M. A. Gribelyuk, J. Li, P. Ronsheim, J. S. Murray, and D. J. Smith, Appl. Phys. Lett. 80, 3213 (2002).

${ }^{12}$ D. Cooper, R. Truche, and J.-L. Rouviere, Ultramicroscopy 108, 488 (2008).

${ }^{13}$ D. Cooper, F. Bertin, P. Salles, and G. Benassayag, Appl. Phys. Lett. 93, 043510 (2008).

${ }^{14}$ W. D. Rau, P. Schwander, F. H. Baumann, W. Hoppner, and A. Ourmazd, Phys. Rev. Lett. 82, 2614 (1999).

${ }^{15}$ International Technology Roadmap for Semiconductors, 2005 ed. (Semiconductor Industry Association, San Jose, CA, 2005), http://public.itrs.net.

${ }^{16}$ K. H. Park, Mater. Res. Soc. Symp. Proc. 199, 271 (1990).

${ }^{17} \mathrm{H}$. Lichte, Ultramicroscopy 108, 256 (2008).

${ }^{18}$ D. Cooper, R. Truche, P. Rivallin, J. Hartmann, F. Laugier, F. Bertin, and A. Chabli, Appl. Phys. Lett. 91, 143501 (2007).

${ }^{19}$ D. Cooper, R. Truche, A. C. Twitchett-Harrison, R. E. Dunin-Borkowski, and P. A. Midgley, J. Microsc. 223, 102 (2009).

${ }^{20}$ S. M. Sze, Semiconductor Devices, Physics and Technology (Wiley, New York, 2002), pp. 180-184.

${ }^{21}$ D. Cooper, A. C. Twitchett, P. A. Midgley, and R. E. Dunin-Borkowski, J. Appl. Phys. 101, 094508 (2007).

${ }^{22}$ M. Beleggia, P. F. Fazzini, P. G. Merli, and G. Pozzi, Phys. Rev. B 67, 045328 (2003).

${ }^{23}$ P. F. Fazzini, P. G. Merli, G. Pozzi, and F. Ubaldi, Phys. Rev. B 72, 085312 (2005).

${ }^{24} \mathrm{http}: / /$ www.silvaco.com.

${ }^{25}$ A. C. Twitchett, R. E. Dunin-Borkowski, R. J. Hallifax, R. F. Broom, and P. A. Midgley, J. Microsc. 214, 287 (2003). 